\title{
Nature de la précipitation dans un alliage hypo-eutectoide de type $\mathrm{Cu}-\mathrm{Al}-\mathrm{Ni}$
}

\author{
S.M.Chentouf ${ }^{\text {a,b }}$, M.Bouabdallah ${ }^{\text {c }}$, N.David ${ }^{a}$, H.Cheniti ${ }^{\text {b,c }}$, J-M.Fiorani $^{\text {a }}$ \\ ${ }^{a}$ Institut Jean Lamour, UMR 7198 CNRS-Université Henri Poincaré Nancy-1, 54506 Vandœuvre-Lès- \\ Nancy, France \\ ${ }^{b}$ Département de Science des Matériaux, Faculté de Génie Mécanique et de Génie de Procédés, USTHB, \\ B.P.32 El-Alia Bab-Ezzour, Alger, Algérie \\ ${ }^{c}$ Laboratoire de Génie Sismique et Dynamique des Structures, E.N.P, 10 Avenue Hassen Badi El-Harrach, \\ Alger, Algérie \\ chent s@yahoo.com \\ mabrouk.bouabdallah@mail.enp.edu.dz \\ Nicolas.david@Icsm.uhp-nancy-.fr \\ cheniti hicham@yahoo.fr \\ Jean-marc.fiorani@Icsm.uhp-nancy.fr
}

\begin{abstract}
Résumé
Notre but est d'étudier la nature et le mécanisme de la précipitation, ayant lieu dans un alliage à mémoire de forme hypo-eutectoïde de type Cu-Al-Ni. A partir d'un état hors équilibre biphasé ( $\alpha$ sursaturé + martensite $\left.\beta^{\prime}\right)$, nous avons réalisé deux cycles successifs de chauffage/refroidissement à la vitesse de $5^{\circ} \mathrm{C} / \mathrm{min}$, entre la température ambiante et $650^{\circ} \mathrm{C}$. Les techniques de caractérisation utilisées (DSC, DRX et MET) ont permis de mettre en évidence que le mécanisme de précipitation est continu et que les précipités stables formés sont de type AINi de forme arrondie avec une taille de l'ordre de $40 \mathrm{~nm}$.
\end{abstract}

\footnotetext{
Mots clés:

Transitions de phases, Intermétalliques, Précipités nanométriques, Alliages à mémoire de forme, Transformation martensitique, DSC (calorimétrie différentielle à balayage)
}

\section{INTRODUCTION}

Les alliages à mémoire de forme sont divisés en famille à savoir : base cuivre, base fer, Ag-Cd, Au-Cd, Ti-Ni, etc ... Les alliages $\mathrm{Ti}-\mathrm{Ni}$ présentent un grand potentiel d'application en raison de leurs excellentes propriétés mécaniques, de la bonne stabilité de leurs propriétés mémoire de forme, mais surtout en raison de leur compatibilité biologique (exemple dans le cas des pathologies de scoliose). Cependant, ces matériaux sont trop coûteux. Les alliages cuivreux et particulièrement les composés du système Cu-Al-Ni possèdent des propriétés thermoélastiques dites hautes températures comparées aux autres types d'alliages. Ils sont de ce fait, un bon compromis entre coût et propriétés.

Dans le domaine riche en cuivre, l'alliage Cu-Al exhibe une transformation eutectoïde pour $11,2 \%$ en poids d'aluminium avec un domaine monophasé en forme de "V" [1], correspondant au domaine d'apparition de la phase $\beta$ haute température de structure cubique centré désordonné. De part et d'autre de ce domaine se trouve la phase $\alpha$ (pour une teneur en aluminium inférieure au point de composition eutectoïde) et la phase $\gamma_{2}$ (pour une teneur en aluminium supérieure au point de composition eutectoïde) dont la précipitation est responsable de l'instabilité thermique de la phase $\beta$ aux températures supérieures à $220^{\circ} \mathrm{C}[2,3]$. La phase $\beta$ haute température subira une transition d'ordre, au cours d'un refroidissement réalisé à une vitesse assez élevée [4] bien supérieure à $2^{\circ} \mathrm{C} / \mathrm{min}$ et qui donnera alors la transformation, $\beta$ désordonnée $\rightarrow \beta_{1}$ ordonnée, et cela à une température inférieure à celle du palier eutectoïde $\left(565^{\circ} \mathrm{C}\right)$. A son tour, la phase métastable $\beta_{1}$ se transformera en phase martensitique au cours d'un refroidissement, jusqu'à la température ambiante, pour donner lieu à l'apparition de différentes sortes de martensite $\left(\beta^{\prime}, \beta^{\prime}{ }_{1}\right.$ et $\left.\gamma^{\prime}{ }_{1}\right)$ dont le domaine d'existence sera fonction de la composition chimique de l'alliage. L'addition d'un troisième élément (nickel) aura pour effet de déplacer la composition du point eutectoïde vers des concentrations plus élevées en aluminium où existe la phase d'équilibre $\gamma_{2}$. Le nickel a également pour effet d'affecter les domaines d'apparition des différentes sortes de martensite $[5,6]$.

Un dispositif en AMF (alliage à mémoire de forme) perd progressivement ses caractéristiques lorsqu'il subit une surchauffe accidentelle ou lors de vieillissements. Ainsi, l'une des principales raisons de la détérioration de l'effet mémoire de forme est le phénomène de précipitation. Le but de ce travail est d'étudier la nature et le mécanisme de la précipitation, ayant lieu dans un alliage à mémoire de forme hypo-eutectoïde de type $\mathrm{Cu}-\mathrm{Al}-\mathrm{Ni}$ au cours de deux cycles de chauffage/refroidissement de la température ambiante à $650^{\circ} \mathrm{C}$ et inversement.

\section{METHODES EXPERIMENTALES}

Un alliage polycristallin de composition nominale en poids Cu- $11,25 \%$ Al- 4,07 \% Ni a été obtenu par le procédé de fusion classique, sous atmosphère contrôlée d'argon, en utilisant un four à induction de moyenne fréquence de marque Leybold. L'alliage obtenu, de $100 \mathrm{~mm}$ de longueur et de $12 \mathrm{~mm}$ de diamètre, a subi un traitement d'homogénéisation de 20 heures à la température de $900^{\circ} \mathrm{C}$ - à l'air. Plusieurs échantillons d'un même lingot ont été découpés à l'aide d'une microtronçonneuse. Ces échantillons ont été par la suite recuits à la température de $850^{\circ} \mathrm{C}$ pendant 15 minutes pour subir ensuite une trempe à l'eau (température ambiante), dans le but d'obtenir la structure hors équilibre attendue. Cette structure a d'ailleurs été révélée par microscopie optique et diffraction des rayons $X(D R X)$.

Les échantillons hors équilibre ont subi deux cycles de chauffage/refroidissement sous atmosphère contrôlée d'argon à l'aide d'un appareil de type DSC 111 (SETARAM) : chauffage de la température ambiante à

This is an Open Access article distributed under the terms of the Creative Commons Attribution-Noncommercial License (http://creativecommons.org/licenses/by-nc/3.0/), which permits unrestricted use, distribution, and reproduction in any noncommercial medium, provided the original work is properly cited. 
$650^{\circ} \mathrm{C}$ à la vitesse de traitement de $5^{\circ} \mathrm{C} / \mathrm{min}$ et maintien de 10 minutes à $650^{\circ} \mathrm{C}$, puis refroidissement jusqu'à la température ambiante. Des caractérisations par diffraction des rayons $X$ en température ont été réalisées à l'air en utilisant un diffractomètre PHILIPS X'pert-Pro utilisant la radiation $\mathrm{K} \alpha$ du cuivre $(\lambda=1,542 \AA)$. Pour l'acquisition des clichés de DRX à une température donnée, un temps de maintien de 10 minutes a été nécessaire. La vitesse de traitement utilisée en DRX que cela soit au cours du chauffage ou au cours du refroidissement est similaire à celle du traitement réalisée en $\mathrm{DSC}\left(5^{\circ} \mathrm{C} / \mathrm{min}\right)$.

Une étude par microscopie électronique en transmission (MET) a été réalisée en utilisant un microscope de marque Philips CM20 opérant sous une tension de 200 $\mathrm{kV}$. Les lames obtenues après polissage mécanique ont ensuite été amincies par attaque électrochimique dans une solution comportant $20 \mathrm{CC}$ acide perchlorique et 400 $\mathrm{CC}$ d'acide acétique (<=> à $5 \% \mathrm{HClO}_{4}+95 \% \mathrm{HCH}_{3} \mathrm{CO}_{2}$ ).

\section{RESULTATS ET DISCUSSIONS}

\subsection{Etude de la structure de trempe}

L'échantillon hors équilibre présente une structure biphasée formée de la phase $\alpha$ sursaturée et de la phase martensitique de type $\beta^{\prime}$ (Fig. 1 et 2). La nature de cette martensite est en accord avec celle révélée par Bouabdallah et al. [4].

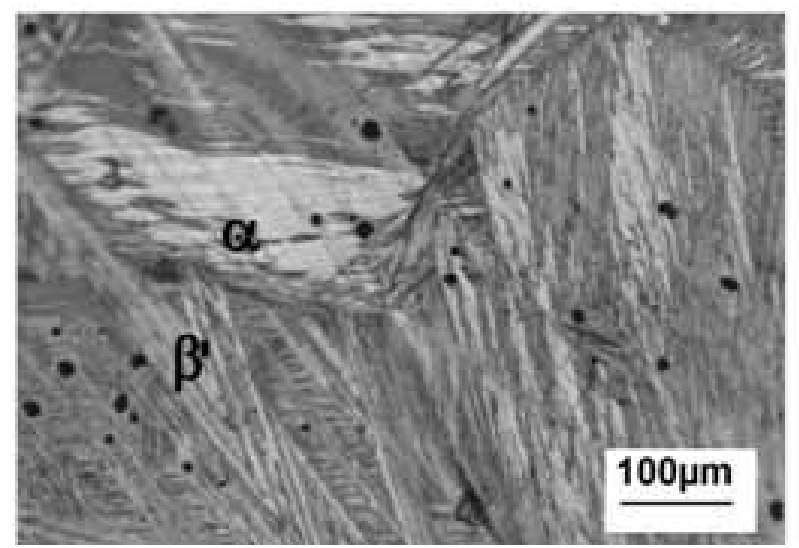

Fig.1 : Micrographie optique de la structure de trempe [7]

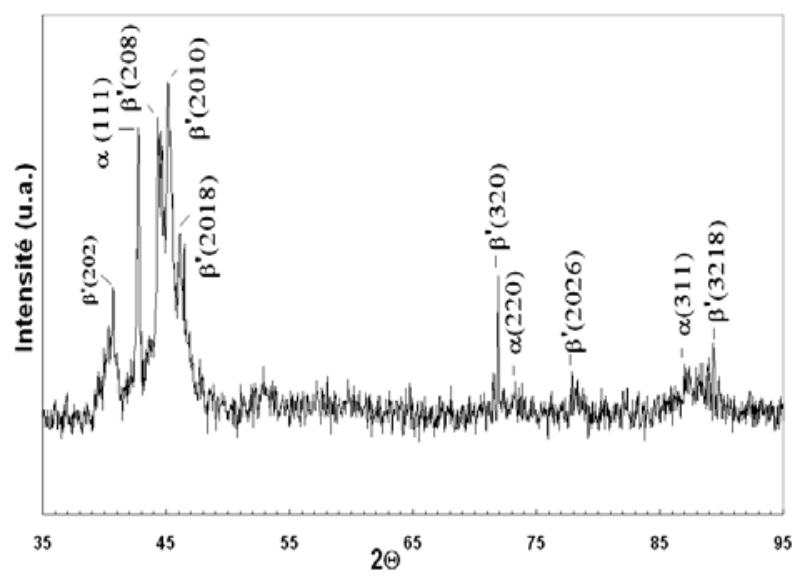

Fig.2 : Diffractogramme RX de la structure de trempe

\subsection{Etude de l'évolution microstructurale au cours du} premier cycle de traitement

L'étude de l'évolution microstructurale réalisée au cours du premier cycle de chauffage/ refroidissement, à la vitesse de $5^{\circ} \mathrm{C} / \mathrm{min}$, montre dans le cas du chauffage (Fig. 3) l'apparition de plusieurs transformations.

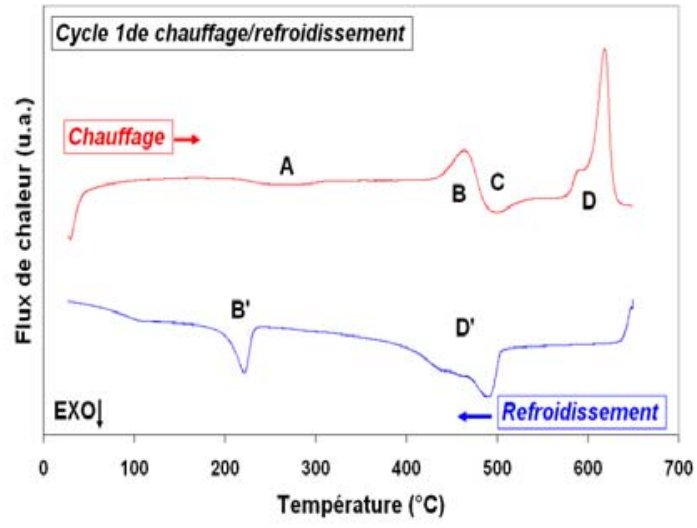

Fig.3: Thermogrammes du premier cycle de chauffage/refroidissement

Ainsi, on voit aux environs de $220^{\circ} \mathrm{C}$ l'apparition d'une évolution $(A)$ de type exothermique correspondant à un domaine de précipitation, d'une phase riche en aluminium et en nickel, par un mécanisme de type continu. Cette évolution est suivie par la transformation (B) qui correspond à la réversion de la phase martensitique $\beta^{\prime}$ en phase $\beta_{1}$ métastable. La transformation suivante dénommée $(\mathrm{C})$ correspond, quant à elle, à la formation des phases d'équilibre $\left(\alpha+\gamma_{2}\right)$. Une partie de la phase d'équilibre $\alpha$ va se recombiner (domaine $D$ ), pour former la phase d'équilibre haute température $\beta$. Ce domaine (D) correspondra également à la dissolution de la phase AINi précédemment formée.

Deux transformations sont observées au cours du refroidissement (Fig. 3). La première évolution dénommée $\left(\mathrm{D}^{\prime}\right)$ correspond d'une part à la décomposition partielle de la phase $\beta$ en constituant d'équilibre $\left(\alpha+\gamma_{2}\right)$ et d'autre part à la précipitation de la phase AINi. La seconde transformation $\left(\mathrm{B}^{\prime}\right)$ correspond à la transformation de la phase $\beta$, non décomposée, en martensite.

Les diffractogrammes du premier cycle de chauffage et de refroidissement (Fig. 4) montrent dans le cas du chauffage (a) à la température de $550^{\circ} \mathrm{C}$ les pics relatifs aux phases $\alpha$ (avec une importante intensité), $\gamma_{2}$ et AINi. A la température de $640^{\circ} \mathrm{C}$, on observe des raies de diffraction de la phase $\beta$ et de la phase $\alpha$ (domaine biphasé $\mathrm{D})$. Au refroidissement (b), il apparaît à $380^{\circ} \mathrm{C}$ (après le domaine $D^{\prime}$ ) les raies des phases $\alpha, \gamma_{2}$, AINi et de la phase $\beta$. Ainsi, la phase $\beta$ se transformera en phase martensitique de type $\beta^{\prime}$ avec pour raie principale celle correspondant au plan (208). 


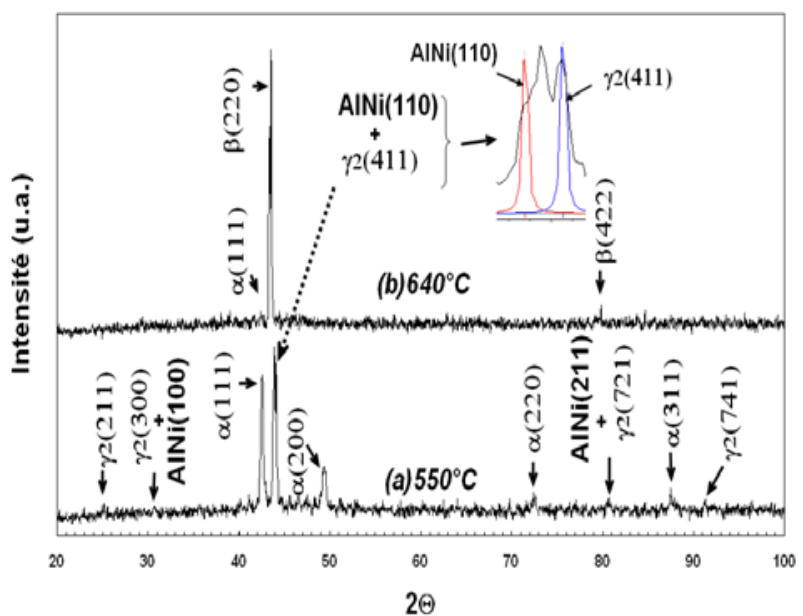

(a)

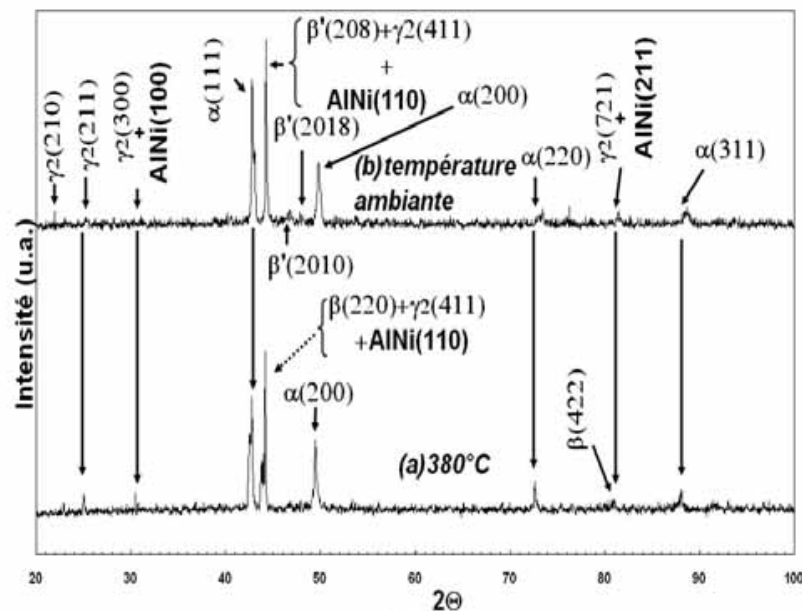

(b)

Fig.4 : Diffractogrammes RX du premier cycle de traitement, (a) chauffage et (b) refroidissement

\subsection{Etude de l'évolution microstructurale au cours du second cycle}

Les thermogrammes du second cycle de chauffage/refroidissement réalisés à la vitesse de $5^{\circ} \mathrm{C} / \mathrm{min}$ (Fig. 5) montrent, au cours du chauffage, la disparition de l'évolution $(A)$ correspondant à la précipitation de la phase AINi. Les transformations (B) et (C) correspondant, respectivement, à la réversion de la phase martensitique $\beta^{\prime}$ en phase $\beta_{1}$ et à la formation des phases d'équilibre ( $\alpha$ $+\gamma_{2}$ ) apparaissent plus étalées. On voit également l'apparition d'une nouvelle transformation dénommée (d). La transformation (d) correspond à l'apparition d'un domaine triphasé formé des phases $(\alpha+\beta+\mathrm{AINi})$. Ce domaine est suivi par la transformation (D) relative au domaine biphasé $(\alpha+\beta)$. Au refroidissement, les transformations sont similaires à celles du premier cycle. Les diffractogrammes de chauffage (Fig. 6a) montrent l'ensemble des phases citées précédemment. Le diffractogramme relatif au refroidissement (Fig. 6b) montre des raies correspondant à la phase hors équilibre (martensite $\beta^{\prime}$ ) ainsi que celles des phases AINi, $\alpha$ et $\gamma_{2}$.

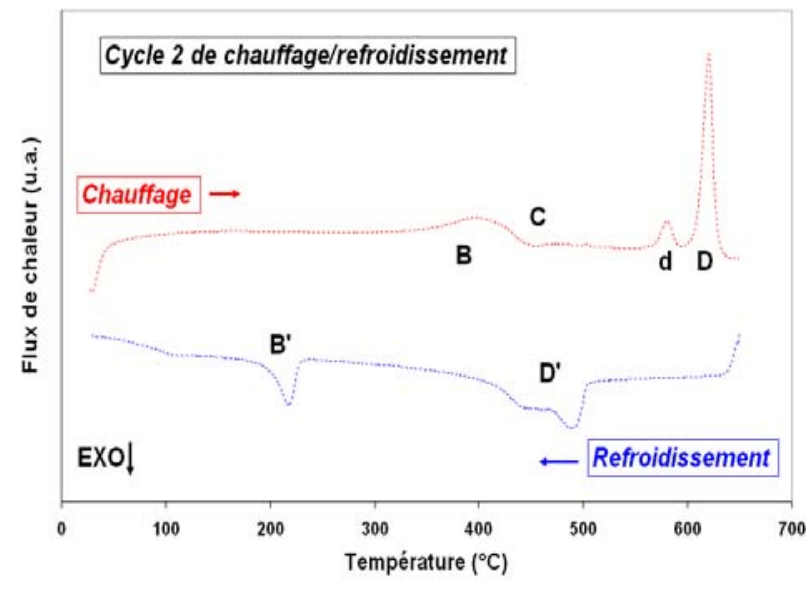

Fig.5: Thermogrammes du second cycle de chauffage/refroidissement

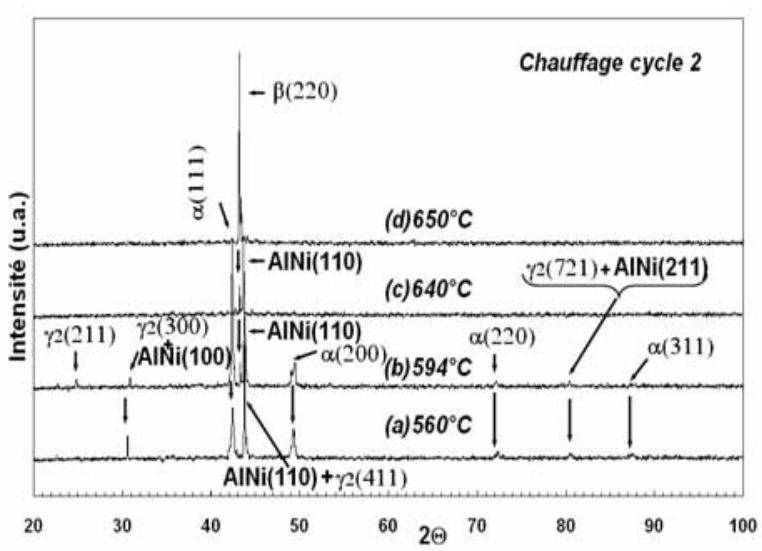

(a)

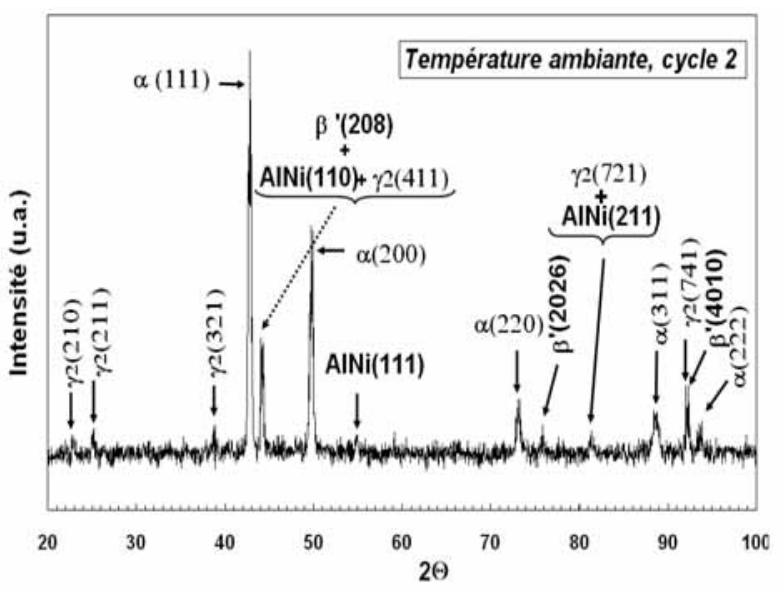

(b)

Fig.6 : Diffractogrammes RX du second cycle de traitement, (a) chauffage et (b) refroidissement

II apparaît à travers les deux cycles de chauffage/refroidissement la présence de précipités $\mathrm{AINi}$ très stables. De même, l'observation micrographique, réalisées par MET (Fig. 7), de la structure suite aux deux cycles de traitement, en accord avec les résultats des analyses par DRX, montre clairement des précipités, de taille nanométrique, de type AINi au sein d'une matrice formée de la phase martensitique $\beta^{\prime}$. Ces précipités sont de forme arrondie avec une taille voisine de $40 \mathrm{~nm}$ (Fig. 7). Ainsi, du fait de leur faible taille, il semble qu'ils 
perturbent peu les plaquettes de martensite en croissance $[7,8]$.

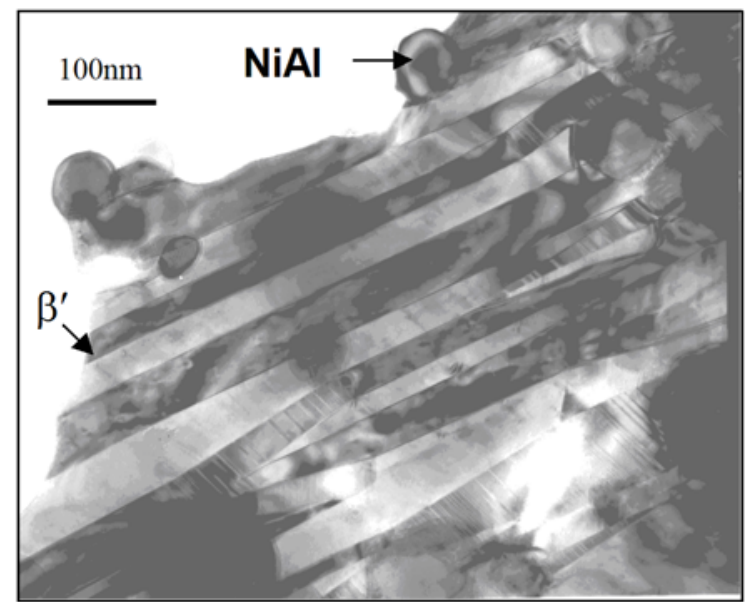

(a)

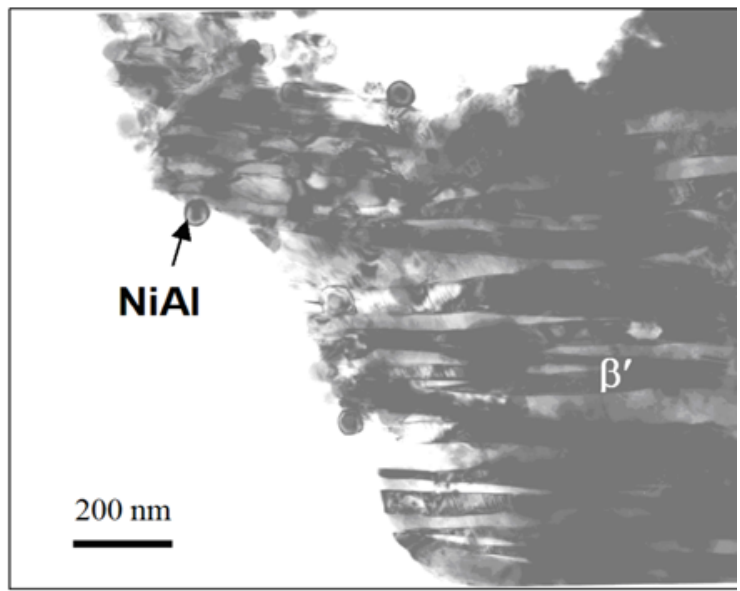

(b)

Fig.7 : Micrographies en microscopie électronique en transmission de l'alliage étudié,

(a) X115000 [7] et (b) X50000

\section{CONCLUSIONS}

Il est apparu à travers cette étude les points suivants :

(a) Apparition par un mécanisme de type continu de précipités de taille nanométrique, de type $\mathrm{AINi}$, très stables.

(b) L'effet d'un second chauffage à la vitesse de $5^{\circ} \mathrm{C} / \mathrm{min}$ montre la disparition de la transformation (A) correspondant à la précipitation de la phase AINi.

\section{REFERENCES}

[1] Murray J.L., 1990, Al-Cu (Aluminium-Copper) in Binary alloy phase diagrams, T.B. Massalski ed., American Society for Metals, Metals Park Ohio, U.S.A.

[2] Recarte V., Pérez-Landazábal J.I., Campo J., PérezSáez R.B., Nó M.L., San Juan J., 2003, J. Phys.IV 112, 605.

[3] Pérez-Landazábal J.I., Recarte V, Nó M.L., San Juan J., 2004, Neutron diffraction analysis of the $\beta$ decomposition process in a texture free $\mathrm{Cu}-\mathrm{Al}-\mathrm{Ni}$ shape memory alloy, Physica B, 350, e1007-e1009.

[4] Bouabdallah M., Cizeron G., 1998, Caractérisation des changements de phase développés dans un alliage
AMF du type Cu-Al-Ni, par dilatométrie de trempe et microcalorimétrie différentielle, Eur. Phys. J. AP 1. 163172.

[5] Bouabdallah M., Cizeron G., 1997, Study of the quenched structure and of the transformation sequences during a slow heating of shape memory Cu-Al-Ni alloys, Ann. Chim. (Paris), 22, 177-182.

[6] Chentouf S.M., Bouabdallah M., 2004, Etude de l'influence de l'aluminium sur l'évolution microstructurale d'alliages à mémoire de forme de type $\mathrm{Cu}-\mathrm{Al}-\mathrm{Ni}, \mathrm{CIMA}^{\prime} \mathrm{O} 4$ Conférence Internationale de Mécanique Avancée, décembre, Boumerdes-Algérie.

[7] Chentouf S.M., Bouabdallah M., Gachon J-C., Patoor E., Sari A., 2009, Microstructural and thermodynamic study of hypoeutectoidal $\mathrm{Cu}-\mathrm{Al}-\mathrm{Ni}$ shape memory alloys, J. Alloys Comp. 470, 507-514.

[8] Bouabdallah M., Baghenane G., Chentouf S.M. Cheniti H., 2007, Influence des traitements de vieillissements en phase $\beta_{1}$ sur le comportement thermoélastique de la transformation martensitique des alliages à mémoire de forme $\mathrm{Cu}-\mathrm{Al}-\mathrm{Ni}$, The Arab Congress on Material Science (ACMS), Gabes-Tunisie. 